\title{
Low Frequency Ultrasonication Induced Antitumor Effect in 5-Aminolevulinic Acid Treated Malignant Glioma
}

\author{
Fumio Yamaguchi $^{{ }^{*}}$, Takayuki Asakura ${ }^{1}$, Hiroshi Takahashi ${ }^{1,2}$, Takayuki Kitamura ${ }^{1}$, \\ Akira Teramoto ${ }^{1,3}$ \\ ${ }^{1}$ Department of Neurosurgery, Nippon Medical School, Tokyo, Japan; ${ }^{2}$ Department of Neurosurgery, Kasugai Rehabilitation Hospi- \\ tal, Yamanashi, Japan; ${ }^{3}$ Tokyo Rosai Hospital, Tokyo, Japan. \\ Email: *fyamaguc@nms.ac.jp
}

Received January $2^{\text {nd }}, 2013$; revised February $1^{\text {st }}, 2013$; accepted February $9^{\text {th }}, 2013$

\begin{abstract}
We investigated the feasibility of sonodynamic therapy for glioma by low frequency ultrasound with 5 -aminolevulinic acid (5-ALA), a precursor of protoporphyrin IX (PpIX) in heme synthetic process. In vivo tumor model was made by inoculating human glioma cell line U87-MG subcutaneously in nude mice. The tumor was sonicated by $25-\mathrm{kHz}$ ultrasound 4 hours following administration of 5-ALA. The tumor size decreased in 5-ALA administered (ALA(+)US(+)) mice, while increased in non-5-ALA administrated (ALA(-)US(+)) mice and non-sonicated mice (ALA(+)US(-)). The immunohistochemical analysis revealed an apoptotic change in tumor tissue of ALA $(+) \mathrm{US}(+)$ mice. The results showed the therapeutic effect of $25 \mathrm{kHz}$ ultrasound for the glioma in 5-ALA administered tumor-bearing mice by inducing apoptotic change of tumor cells. This is a first report to elucidate the feasibility of therapeutic use of $25 \mathrm{kHz}$, relatively low frequency, ultrasound in sonodynamic therapy using 5-ALA as a sonosensitizer precursor. The utilization of this frequency will contribute to the development of sonodynamic therapy for gliomas and the spread of this technique in many hospitals that possess ultrasonic aspirators.
\end{abstract}

Keywords: Glioma; Sonodynamic Therapy; 5-Aminolevulinic Acid; Protoporphyrin IX; Ultrasonication

\section{Introduction}

Glioma has an invasive nature and often invades into the proximity of critically functioning structures. Gross total resection, even though it is a standard therapy, often results in postoperative neurological deteriorations. To avoid this sequel, photodynamic therapy is a promising technique to kill tumor tissue selectively near the functioning brain tissue. Although photodynamic therapies have been investigated and reported to be effective therapies for a wide variety of cancers [1], this technique failed to kill glioma cells locating deep in the brain because of low penetration of light in the brain tissue.

Ultrasound, which has a property of conducting into deep tissues, activates sonsensitizers and induces their cytotoxic effects. Protoporphyrin IX (PpIX), metabolic product of 5-aminolevulinic acid (5-ALA), is known as a sonodynamically activated sensitizer as well as a photosensitizer [2-4]. Because 5-ALA has been widely used for photo diagnosis to confirm the existence of residual tumor tissue during brain tumor resection [5], 5-ALAderived PpIX seems a good candidate as a safe sonosen-

${ }^{*}$ Corresponding author. sitizer in brain tumors. Also PpIX has a property of tumor-specific accumulation [6]. Therefore it is important to know whether sonodynamic therapy (SDT) in combination with 5-ALA shows significant in vivo antitumor effects in gliomas. In most studies, the ultrasound frequency above $1 \mathrm{MHz}$ is used in sonodynamic therapy to activate sonosensitizers $[7,8]$. In this study we investigated the feasibility of sonodynamic therapy with relatively low frequency ultrasound of $25 \mathrm{kHz}$ which is often used in ultrasonic surgical aspirators $[9,10]$.

This is a first report on the utilization of low-frequency ultrasound for sonodynamic therapy of human glioma. And the utilization of this frequency will contribute to the development of sonodynamic therapy for gliomas and the spread of this technique in many hospitals that possess ultrasonic aspirators.

\section{Materials and Methods}

\subsection{Chemical}

5-aminolevulinic acid (Cosmo BioCo., Ltd., Tokyo, Japan) was dissolved in $1 \mathrm{~N} \mathrm{HCl}$ solution and $100 \mathrm{mM}$ stock solution was sterilized, aliquoted and stored in the 
dark at $-20^{\circ} \mathrm{C}$.

\subsection{Glioma Cell Line}

U87MG is a well-characterized human glioblastoma cell line [11]. Cells were cultured in Dulbecco's modified Eagle's medium (DMEM, Gibco, Life Technologies, Inc., USA) supplemented with $10 \%$ fetal bovine serum (FBS, Hyclone, USA), $100 \mathrm{U} / \mathrm{ml}$ penicillin and $100 \mu \mathrm{g} / \mathrm{ml}$ streptomycin at $37^{\circ} \mathrm{C}$ in a humidified atmosphere of $5 \%$ $\mathrm{CO}_{2}$ atmosphere. Cells in the exponential phase of growth were used in each experiment.

\subsection{Ultrasonic Generator}

The ultrasound transducer was manufactured by TOKYO IKEN CO., LTD (Tokyo, Japan). Its ultrasonic electronic generator generates a $25 \mathrm{kHz}$ signal, which is converted by the transducer to a mechanical vibration of the internal piezoelectric crystals. An ultrasound transducer with a diameter of $10 \mathrm{~mm}$ was used with a resonant frequency of $25 \mathrm{kHz}$. The vibration is amplified and transmitted down the length of the probe where the tip longitudinally expands and contracts. The distance the tip travels is dependent on the amplitude. To specify the intensity in the insonation experiment, the reading power $(\mu \mathrm{m})$ of generator was used which represented the amplitude of vibrating width of the tip of transducer.

\subsection{Animals}

The virgin female nu/nu BALB/c mice (5 weeks old; 19 $\pm 2 \mathrm{~g}$ ) used in these experiments were obtained from Charles River Laboratories Japan, INC.

Mice were housed in micro isolation in a dedicated, pathogen-free facility, and all animal experimentation was conducted in accordance with the declaration of Helsinki or the Guide for the Care and Use of Experimental Animals of Nippon Medical School.

\subsection{In Vivo Tumor Model}

Twenty one mice were subcutaneously inoculated with U87-MG tumor cells $\left(1 \times 10^{7}\right.$ cells/mouse, respectively $)$ in the right legs. All tumors reached to the target volume by approximately four weeks after inoculation.

\subsection{Treatment in Nude Mice}

The 20 tumor-bearing mice were randomly divided into four groups: control group (ALA(-)US(-)), ALA only group (ALA(+) US(-)), sonication only group (ALA(-)$\mathrm{US}(+))$, and ALA plus sonication group (ALA $(+) \mathrm{US}(+))$. Each group had five mice. 5-ALA was administered to the mice of ALA $(+) \mathrm{US}(-)$ and $\mathrm{ALA}(+) \mathrm{US}(+)$ groups at a dose of $100 \mathrm{mg} / \mathrm{kg}$ via transgastric route.

\subsection{Confirmation of Protoporphyrin IX Expression}

The surface of the subcutaneous tumors were exposed by skin opening and irradiated by $405 \mathrm{~nm}$ blue Lasers generated by diode Lasers apparatus manufactured for experimental use byIHI Corporation (Tokyo, Japan). Protoporphyrin IX expression was confirmed by the observation of emitting red light.

\subsection{Ultrasound Exposure}

Animals were anesthetized with $50 \mathrm{mg} / \mathrm{kg}$ ketamine and $10 \mathrm{mg} / \mathrm{kg}$ xylazine after four-hour incubation with 5ALA. An acoustically transparent gel was applied on the skin over the subcutaneous tumor. An ultrasound transducer with a diameter of $10 \mathrm{~mm}$ was horizontally attached on the surface of skin over subcutaneous tumor. The tumors were sonicated using ultrasound of $25 \mathrm{kHz}$ at an intensity of $4 \mu \mathrm{m}$ for 4 minutes individually for five mice of ALA $(+) \mathrm{US}(+)$ group and five mice of $\mathrm{ALA}(-)$ $\mathrm{US}(+)$ group. Five mice of ALA(-)US(-) and five mice of ALA(+)US(-) groups were not sonicated. These experiments were conducted at one sonication condition based on the experiments showing it was the most efficient conditions for in vivo sonodynamic treatment with this apparatus. All experiments were conducted at $20^{\circ} \mathrm{C}$ under a dim light condition.

\subsection{Tumor Growth Analysis}

Tumor sizes were measured with calipers for each individual mouse once a week, and the tumor volume $\left(\mathrm{mm}^{3}\right)$ was calculated as $(\mathrm{L} \times \mathrm{W} \times \mathrm{H}) / 2$, where $\mathrm{L}=$ length $(\mathrm{mm})$, $\mathrm{W}=$ width $(\mathrm{mm})$ and $\mathrm{H}=$ height $(\mathrm{mm})$. Growth rates were calculated; Tumor growth rate $=$ tumor volume $\left(\mathrm{mm}^{3}\right)$ of the each time point /tumor volume $\left(\mathrm{mm}^{3}\right)$ at the sonication, and plotted ( \pm Standard error of mean: SE).

\subsection{Statistical Analysis}

Mann-Whitney's U test was performed to determine differences of tumor growth rate between treatment groups. A $P$ value of $<0.05$ was considered significant.

\subsection{Histological Analysis and Evaluation of Apoptosis}

One tumor-bearing mouse was prepared to analyze the histological change after ultrasonic treatment. Subcutaneous tumor was treated by ultrasonication under the same experimental condition as ALA $(+)$ group, but when the tumor size reached at the approximately $15 \mathrm{~mm}$ in diameter. One week after treatment, a mouse was sacrificed by cervical dislocation and the tumors were removed. A tumor sample was fixed in $10 \%$ formaldehyde 
for 6 hours and paraffin embedded. Thin sections of 5 microns were stained with hematoxylin-eosin (H\&E). Tissue section was also treated using ApopTag ${ }^{\circledR}$ (Millipore, MA, USA) for the detection of fragmented genomic DNA. Briefly, paraffin sections were dewaxed and rehydrated, treated with proteinase $\mathrm{K}(20 \mu \mathrm{g} / \mathrm{ml})$ for 15 min and washed in PBS. Endogenous peroxidase was blocked with $3 \% \mathrm{H}_{2} \mathrm{O}_{2}$ for 15 min. Slides were then covered with equilibration buffer and were incubated for 1 $\min$ at $37^{\circ} \mathrm{C}$. This was followed by the incubation with the $\mathrm{TdT}$ for $60 \mathrm{~min}$ at $37^{\circ} \mathrm{C}$. The reaction then was terminated using the ApopTag ${ }^{\circledR}$ stop buffer followed by anti-digoxigenin-peroxidase application. Fluorescence was viewed by fluorescence microscopy using standard fluorescein excitation and emission long pass filters for FITC.

\section{Results}

\subsection{SDT Induced Suppression of Tumor Growth}

In vivo, the size of tumors decreased in the ALA $(+) \mathrm{US}(+)$ mice, while that of $\operatorname{ALA}(-) \mathrm{US}(-), \operatorname{ALA}(-) \mathrm{US}(+)$ and ALA $(+)$ US(-) group showed the continuous growth of tumors. In Figure 1, representative animals of ALA $(+)-$ $\mathrm{US}(+)$ group and ALA(-)US(+) group were shown. The subcutaneous tumor was rapidly shrinking and diminished by Day21. Tumors volumes of 5-ALA plus sonication group $(\mathrm{ALA}(+) \mathrm{US}(+))$ decreased after transdermal sonication, while tumors of non-5-ALA group (ALA(-)US(+)) were continuously growing (Figure 2). The mean of tumor growth rate of ALA(+)US(+) group was $0.08 \pm$ 0.08 (mean $\pm \mathrm{SE}$ ), while the other groups showed high growth rate; ALA(-)US(-): $6.89 \pm 1.19$, ALA $(+) \mathrm{US}(-)$ : $4.85 \pm 1.69$, and ALA(-)US(+): $5.08 \pm 2.77$ (Figure 2).

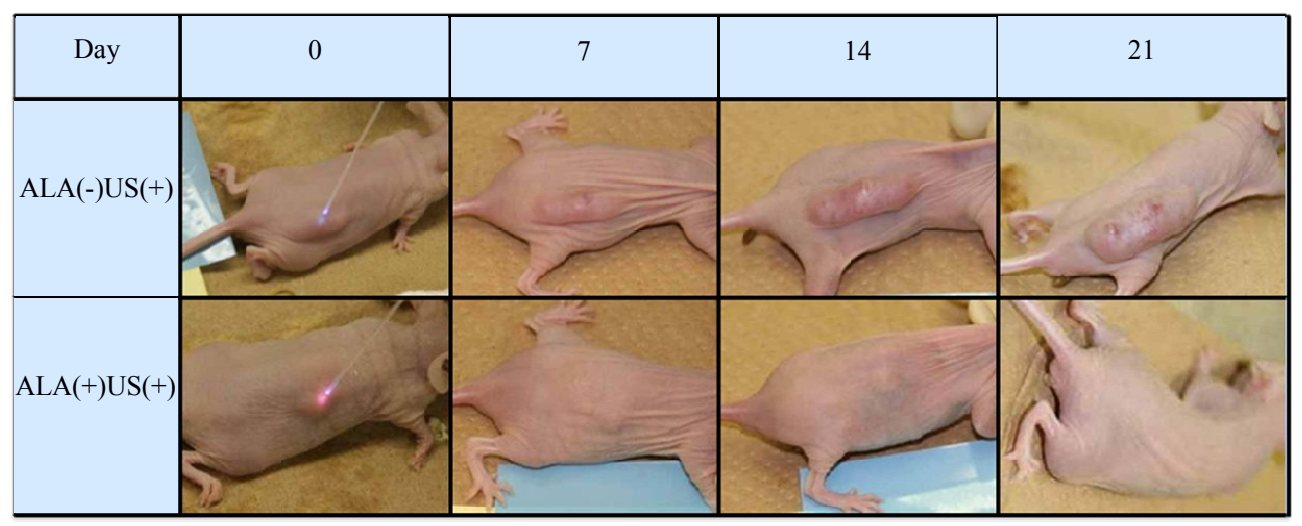

Figure 1. Subcutaneous tumors. Tumors volumes of 5-ALA plus sonication group $(A L A(+) U S(+))$ decreased after transdermal sonication, while tumors of non-5-ALA group (ALA(-)US(+)) were continuously growing. (Representative cases shown).

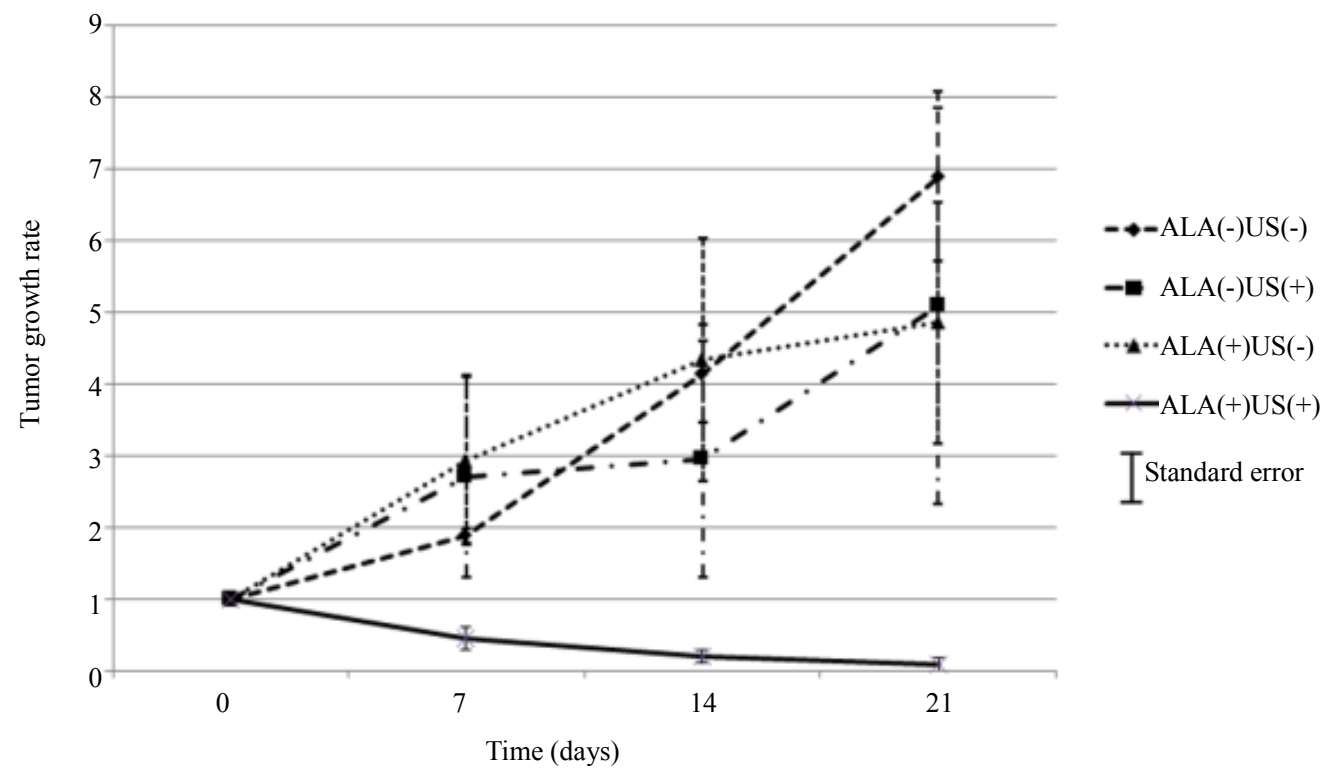

Figure 2. Growth rate of implanted tumors. The tumor growth rate of ALA(+)US(+) group showed significant decrease after sonication, while no decrease in ALA(-)US(-), ALA(-)US(+) and ALA(+)US(-) group. (SE: standard error of mean). 
The decrease of the growth rate of $\operatorname{ALA}(+) \mathrm{US}(+)$ group is statistically significant at 21 days after sonication compared with the other groups; ALA $(-) \mathrm{US}(+)(\mathrm{p}=0.0214)$, $\mathrm{ALA}(+) \mathrm{US}(-)(\mathrm{p}=0.0121)$ and $\operatorname{ALA}(-) \mathrm{US}(-)(\mathrm{p}=$ 0.0121).

Since the growth rate at Day21 of ALA(-)US(+) group was not different from that of ALA(-)US(-) group $(\mathrm{p}=$ 0.529), ultrasonication itself did not show suppressive effect on tumor growth. Also the growth rates of ALA(+)US(-) and ALA(-)US(-) group were not different $(\mathrm{p}=0.095), 5$-aminolevulinic acid was not harmful on tumor at this dose.

\subsection{SDT Induced Apoptosis of Tumor}

H\&E staining revealed that treatment by sonication mediated significant apoptosis in U87MG glioma in vivo (Figures 3(A) and (B)). A broad zone of a nuclear and a cellular eosinophilic debris, ringed by a zone of tumor cells in the process of undergoing apoptosis, was evident in the $\mathrm{ALA}(+) \mathrm{US}(+)$ group but not the other groups. Damaged tumor by this therapy showed the vast apoptosis component to a depth of approximately $1 \mathrm{~cm}$ (Figures 3(C) and (D)), while no apoptotic change was seen in tumor tissue where sonication was not applied.

\section{Discussion}

\subsection{Antitumor Effect}

Sonodynamic therapy is an approach based on the synergistic effect of ultrasound and chemical compound referred to as "sonosensitizer" for cancer treatment. Se-

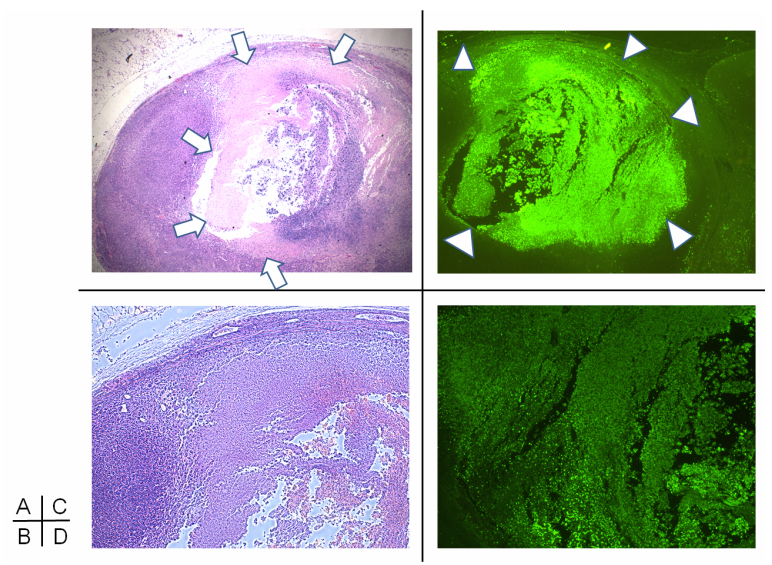

Figure 3. Apoptotic change of tumors at one week after 5-ALAplus ultrasonic irradiation in $\operatorname{ALA}(+) \mathrm{US}(+)$ group. H\&E staining of tumor section (A) and high power magnification (B) shows a broad zone of anuclear and acellular eosinophilic debris (arrows), ringed by a zone of tumor cells in the process of undergoing apoptosis. ApopTag ${ }^{\circledR}$ staining (C) detected fragmented genomic DNA (D: high power magnification) and showed the vast apoptosis component to a depth of approximately $1 \mathrm{~cm}$ (arrow heads). veral studies have been conducted in vitro and in vivo to investigate the cytotoxic effect of the combination of ultrasounds and hematoporphyrin, the most common photodynamic sensitizer, showing encouraging results [12-14]. Recently the in vivo effect of the combination of PpIX and ultrasound exposure on a subcutaneously implanted sarcoma 180 (S180) solid tumor was investigated with ultrasound at a frequency of $2.2 \mathrm{MHz}$ [15]. However, the sonodynamic effect of PpIX on glioma has not been studied in details so far, moreover low frequency as $25 \mathrm{kHz}$ instead of high frequency as $2 \mathrm{MHz}$ is not studied.

Our experiment showed that the increase of tumor volume was remarkably inhibited with combination of 5-ALA and ultrasound. Since the growth rate at Day21 of ALA(-)US(+) group was not different from that of ALA(-)US(-) group, sonication itself did not show any tumor suppressive effect. 5-aminolevulinic acid was also not harmful on tumor at this dose because the growth rates of ALA $(+) \mathrm{US}(-)$ and ALA(-)US(-) group were not statistically different. Therefore tumor suppression may be induced by the synergistic effects of 5-ALA and ultrasound. Since the sonication immediate after administration of 5-ALA did not show any tumor suppressive effect, 5-ALA-derived chemicals, instead 5-ALA itself, may have worked for cytotoxicity. PpIX is one of metabolites of 5-ALA known as a photosensitizer and also a sonosensitizer. The production of PpIX was confirmed by red color photic excitation at the time of sonication, so that PpIX may be the most probable chemical working as a sonosensitizer in our study.

\subsection{The Mechanism of SDT}

Microscopic observation found that the cell ultra-structure was significantly damaged, and apoptotic change of tumor cells remarkably increased after sonodynamic treatment. Recently Song et al. [7] reported that sonodynamic therapy using 5-aminolevulinic acid induced the apoptotic change of SAS human tongue squamous cell carcinoma cells. Also Li et al. [16] also reported the detail tumor cell change that caspase-depended apoptosis could be induced by PpIX-SDT in human breast cancer MDA-MB-231 cells, and the intracellular reactive oxygen species were involved during the apoptotic process. In these reports, $1.05 \mathrm{MHz}$ ultrasound was used which is a popular ultrasound frequency for sonodynamic therapies. Considering the result of our experiment in which the frequency of $25 \mathrm{kHz}$ used, this apoptotic change occurs not only by high frequencies but also by low frequencies around $25 \mathrm{kHz}$.

\subsection{Potential Clinical Application}

Photodiagnosis (PD) using 5-ALA has been one of the 
popular methods to confirm the existence of tumor tissue during the removal or the biopsy of brain tumors [5]. 5-ALA is known as a safe and reliable chemical used clinically in glioma patients and our data showed that it is working in synergy with ultrasound inducing apoptotic change of glioma cells. This method is a promising therapy on the unresectable tumors.

The frequency that we used is the vibration frequency of ultrasound surgical aspirator which is often used in brain tumor surgeries. If this frequency can be used for sonodynamic therapy for glioma, it seems feasible to use the ultrasound surgical aspirator with minimal change such as an exchange of hand piece. Even though this technique is a promising therapy, its clinical applications are still under debate since the specific mechanisms of sonosensitization are influenced by multiple factors, including the nature of the biological model, the sonosensitizer and the ultrasound parameters.

The optimal conditions to obtain stable antitumor effect should be elucidated before application of this method to clinical cases.

In summary, Low-frequency sonochemical therapy utilizing 5-ALA reduced the volume of malignant gliomas. The mechanism was suggested to be the induction of apoptosis in glioma cells. This therapy may become an alternative treatment for unresectable invaded tumors in patients in whom gross total resection is considered to result in unacceptable sequel. Further analyses are necessary to conclude that this sonodynamic therapy can be clinically applied to glioma therapy.

\section{Acknowledgement}

This work was supported in part by a grant of Strategic Research Foundation Grant-aided Project for Private Universities from Ministry of Education, Culture, Sport, Science, and Technology, Japan (MEXT) (S0801035).

Technological supports on ultrasonic device were provided by TOKYO IKEN CO., LTD (Tokyo, Japan).

We are grateful to Mr. Katsushi Inoue, (SBI Pharmaceuticals Co., Ltd., Tokyo, Japan) for his technical support on Lasers apparatus.

\section{REFERENCES}

[1] P. Agostinis, K. Berg, K. A. Cengel, T. H. Foster, A. W. Girotti, S. O. Gollnick, S. M. Hahn, M. R. Hamblin, A. Juzeniene, D. Kessel, M. Korbelik, J. Moan, P. Mroz, D. Nowis, J. Piette, B. C. Wilson and J. Golab, "Photodynamic Therapy of Cancer: An Update," CA: A Cancer Journal for Clinicians, Vol. 61, No. 4, 2011, pp. 250-281. doi:10.3322/caac.20114

[2] Q. Peng, T. Warloe, K. Berg, J. Moan, M. Kongshaug, K. E. Giercksky and J. M. Nesland, "5-Aminolevulinic AcidBased Photodynamic Therapy," Cancer, Vol. 79, No. 12, 1997, pp. 2282-2308.
doi:10.1002/(SICI)1097-0142(19970615)79:12<2282::AI D-CNCR2>3.0.CO;2-O

[3] X. Wang, P. Wang, W. Tong and Q. Liu, "Comparison of Pharmacokinetics, Intracellular Localizations and Sonodynamic Efficacy of Endogenous and Exogenous Protoporphyrin IX in Sarcoma 180 Cells," Ultrasonics, Vol. 50, No. 8, 2010, pp. 803-810. doi:10.1016/j.ultras.2010.04.004

[4] R. Canaparo, L. Serpe, M. G. Catalano, O. Bosco, G. P. Zara, L. Berta and R. Frairia, "High Energy Shock Waves (HESW) for Sonodynamic Therapy: Effects on HT-29 Human Colon Cancer Cells," Anticancer Research, Vol. 26, No. 5A, 2006, pp. 3337-3342.

[5] W. Stummer, U. Pichlmeier, T. Meinel, O. D. Wiestler, F. Zanella, H. J. Reulen and ALA-Glioma Study Group, "Fluorescence-Guided Surgery with 5-Aminolevulinic Acid for Resection of Malignant Glioma: A Randomised Controlled Multicentre Phase III Trial," Lancet Oncology, Vol. 7, No. 5, 2006, pp. 392-401. doi:10.1016/S1470-2045(06)70665-9

[6] Z. Ji, G. Yang, V. Vasovic, B. Cunderlikova, Z. Suo, J. M. Nesland and Q. Peng, "Subcellular Localization Pattern of Protoporphyrin IX is an Important Determinant for Its Photodynamic Efficiency of Human Carcinoma and Normal Cell Lines," Journal of Photochemistry and Photobiology B, Vol. 84, No. 3, 2006, pp. 213-220. doi:10.1016/j.jphotobiol.2006.03.006

[7] W. Song, H. Cui, R. Zhang, J. Zheng and W. Cao, "Apoptosis of SAS Cells Induced by Sonodynamic Therapy Using 5-Aminolevulinic Acid Sonosensitizer," Anticancer Research, Vol. 31, No. 1, 2011, pp. 39-45.

[8] M. Kinoshita and K. Hynynen, "Mechanism of Porphyrin-Induced Sonodynamic Effect: Possible Role of Hyperthermia," Radiation Research, Vol. 165, No. 3, 2006, pp. 299-306. doi:10.1667/RR3510.1

[9] F. Epstein, "The Cavitron Ultrasonic Aspirator in Tumor Surgery," Clinical Neurosurgery, Vol. 31, 1983, pp. 497 505.

[10] M. Brock, I. Ingwersen and W. Roggendorf, "Ultrasonic Aspiration in Neurosurgery," Neurosurgical Review, Vol. 7, No. 2-3, 1984, pp. 173-177. doi:10.1007/BF01780701

[11] G. Beckman, L. Beckman, J. Pontén and B. Westermark, "G-6-PD and PGM Phenotypes of 16 Continuous Human Tumor Cell Lines. Evidence Against Cross-Contamination and Contamination by HeLa Cells," Human Heredity, Vol. 21, No. 3, 1971, pp. 238-241. doi: $10.1159 / 000152408$

[12] A. E. Worthington, J. Thompson, A. M. Rauth and J. W. Hunt, "Mechanism of Ultrasound Enhanced Porphyrincytotoxity. Part I: A Search for Free Radical Effects," Ultrasound in Medicine \& Biology, Vol. 23, No. 7, 1997, pp. 1095-1105. doi:10.1016/S0301-5629(97)00019-7

[13] N. Yumita and S. Umemura, "Sonodynamic Therapy with Photofrin II on AH130 Solid Tumor. Pharmacokinetics, Tissue Distribution and Sonodynamicantitumoral Efficacy Ofphotofrin II," Cancer Chemotherapy and Pharmacology, Vol. 51, No. 2, 2003, pp. 174-178. doi:10.1007/s00280-002-0523-6

[14] N. Mi, Q. Liu, X. Wang, X. Zhao, W. Tang, P. Wang and 
B. Cao, "Induction of Sonodynamic Effect with Protoporphyrin IX on Isolate Hepatoma-22 Cells," Ultrasound in Medicine \& Biology, Vol. 35, No. 4, 2009, pp. 680-686. doi:10.1016/j.ultrasmedbio.2008.07.002

[15] X. B. Wang, Q. H. Liu, P. Wang, W. Tang and Q. Hao, "Study of Cell Killing Effect on S180 by Ultrasound Activating Protoporphyrin IX," Ultrasonics, Vol. 48, No. 2,
2008, pp. 135-140. doi:10.1016/j.ultras.2007.11.001

[16] Y. Li, P. Wang, P. Zhao, S. Zhu, X. Wang and Q. Liu, "Apoptosis Induced by Sonodynamic Treatment by Protoporphyrin IX on MDA-MB-231 Cells," Ultrasonics, Vol. 52, No. 4, 2012, pp. 490-496. doi:10.1016/j.ultras.2011.10.013

\section{Abbreviations}

5-ALA, 5-aminolevulinic acid;

PpIX, protoporphyrin IX;

SDT, sonodynamic therapy 